\title{
Identification of key genes influenced by fixation stability in early fracture hematoma and elucidation of their roles in fracture healing
}

\author{
CHENGXUE WANG $^{1}$, BAOCHANG $^{1}{ }^{1}$, CONGFENG ZHANG $^{2}$ and JIEPING CHENG ${ }^{3}$ \\ ${ }^{1}$ Department of Orthopedic Trauma, The First Hospital of Jilin University, Changchun, Jilin 130021; \\ ${ }^{2}$ Department of Orthopedics, The Second People's Hospital of Yushu; ${ }^{3}$ Department of Orthopedics, \\ The Second Hospital of Jilin University, Changchun, Jilin 130041, P.R. China
}

Received March 7, 2016; Accepted February 24, 2017

DOI: $10.3892 /$ etm.2017.5192

\begin{abstract}
The present study aimed to identify the key genes influenced by fixation stability in early fracture hematoma and to elucidate their roles in fracture healing. The GSE53256 gene expression profile, including six fracture hematoma tissues, was downloaded from the Gene Expression Omnibus database. The differentially expressed genes (DEGs) in the fracture hematoma tissues from old rats with rigid fixation compared with semi-rigid fixation were identified using the limma package. Furthermore, Gene Ontology (GO) enrichment analysis for DEGs was performed using BiNGO, and a protein-protein interaction (PPI) network was constructed based on the Search Tool for the Retrieval of Interacting Genes database. A total of 265 DEGs (158 upregulated and 107 downregulated) in the fracture hematoma tissues were screened out. Additionally, the overrepresented GO terms were mainly associated with the extracellular region, positive regulation of locomotion and response to external stimulus. Transforming growth factor, $\beta 1$ (Tgf $\beta 1)$, chemokine (C-X-C motif) ligand 12 (Cxcl12), matrix metallopeptidase 9 (mmp9) and serpin peptidase inhibitor, clade E, member 1 (serpine1) had higher degrees and were hub nodes in the PPI network. In conclusion, fixation stability may influence the fracture healing process, and important DEGs, including Cxcl12, mmp9, $\operatorname{Tgf} \beta 1$ and serpine1, may be important in this process.
\end{abstract}

Correspondence to: Dr Jieping Cheng, Department of Orthopedics, The Second Hospital of Jilin University, 18 Ziqiang Street, Changchun, Jilin 130041, P.R. China

E-mail: cheeijp@163.com

Key words: fixation stability, fracture hematoma, fracture healing, differentially expressed genes, Gene Ontology enrichment analysis, protein-protein interaction network

\section{Introduction}

Bone regeneration is a cascade of complex biological events of bone induction and translation, and is required to optimize skeletal repair and restore skeletal function $(1,2)$. It has been shown to be involved in numerous conditions, including skeletal reconstruction of large bone defects created by trauma, orthopedic surgery and osteotomy (3). The most common form of bone regeneration is fracture healing in the clinical setting (4). Thus, understanding the mechanism of fracture healing and accelerating the overall regeneration process will help to improve the healing outcome and alleviate the pain felt be patients to some extent.

Fracture consolidation can be influenced by mechanical factors, and mechanical stability is confirmed to be a key factor for determining the healing outcome of bone regeneration $(5,6)$. It has also been demonstrated that the optimal fixation rigidity, which is neither too stiff nor too gentle, is associated with the fastest growth of the bone (7). Ranganathan et al (8) also reported that an appropriate fixation stability was necessary to promote timely fracture healing. In addition, the early fracture hematoma has been shown to have potency and potential in fracture healing and has become a subject of attention (9). An in vitro study has indicated that fracture hematoma contains multilineage mesenchymal progenitor cells able to differentiate into a chondrogenic or osteogenic cell type and thus is important in bone healing (10). Furthermore, fracture hematoma has been shown to contain growth factor vascular endothelial growth factor, which is crucial in angiogenesis and fracture healing $(11,12)$. Sarahrudi et al $(13)$ demonstrated that fracture hematoma had significantly higher concentrations of transforming growth factor, $\beta 1$ (Tgf $\beta 1)$ than the peripheral serum of patients. Therefore, the early fracture hematoma may be important in the early healing period and may determine the healing outcome. Furthermore, gene expression in the early fracture hematoma is also reported to be influenced by fixation stability (14). However, it so far remains incompletely understood what molecules are involved in the early fracture hematoma associated with fixation stability, as well as their roles during fracture healing. 
In a previous study, GSE53256 microarray data was utilized to analyze the interactive effects of age and mechanical stability on bone defect healing using an early transcriptional analysis (14). In the present study, the GSE53256 microarray data was downloaded and a bioinformatics approach was applied in order to identify the differentially expressed genes (DEGs) in fracture hematoma tissues from old rats with rigid fixation in comparison with fracture hematoma tissues from old rats with semi-rigid fixation. In addition, functional enrichment analysis and protein-protein interaction (PPI) network analysis were performed. The aim was to identify the potential key genes influenced by fixation stability in early fracture hematoma, as well as to elucidate their roles in the process of fracture healing.

\section{Materials and methods}

Affymetrix microarray data. The gene expression profile of GSE53256 deposited by Ode et al (14) was obtained from the Gene Expression Omnibus (http://www.ncbi.nlm.nih.gov/geo/) database; the profile was performed on the platform of GPL1335 [Rat 230_2] Affymetrix Rat Genome 2302.0 Array (Affymetrix, Santa Clara, CA, USA). A total of 12 fracture hematoma tissues from four groups of Sprague-Dawley rats with a 1.5-mm osteotomy gap in the femora with varying age (12 vs. 52 weeks) and fixator stiffness (rigid vs. semi-rigid fixation; $n=3$ per group) were used for the development of this microarray data. However, in this study, only the expression data from the six fracture hematoma tissues of the old (52-week-old) rats after 3 days of an osteotomy and with rigid or semi-rigid fixation were used for the analysis.

Data preprocessing and DEG screening. The raw expression data were first background corrected and quantile normalized by the robust multiarray average (15) method with application of the Affy package in R/Bioconductor. Next, the DEGs in fracture hematoma tissues from old rats with rigid fixation compared with fracture hematoma tissues from old rats with semi-rigid fixation were identified using limma (16) package in R/Bioconductor (http://www.bioconductor.org/packages/release/bioc/html/limma.html), where a fold-change $\geq 1.5$ and $\mathrm{P}<0.05$ were defined as the cutoff value. Additionally, hierarchical clustering analysis of the DEGs was performed and visualized using the pheatmap package in $\mathrm{R}(17)$.

Functional enrichment analysis. Gene Ontology (GO; http://www.geneontology.org) (18) is widely applied for the biological unification of large-scale gene lists, which are mainly classified into three categories, namely biological process (BP), molecular function and cellular component. In the present study, GO-BP enrichment analysis for DEGs was performed using the BiNGO (http://www.psb.ugent.be/cbd/papers/BiNGO/) (19) plugin and was then visualized using Cytoscape software (20). In order to reduce false positives, multiple testing correction was performed using the Beniamini-Hochberg method (21), and the P-value was then adjusted as the false discovery rate (FDR). Finally, significant enrichment threshold of GO-BP terms was set as FDR<0.05.
PPI network construction. The Search Tool for the Retrieval of Interacting Genes (STRING; http://string-db.org/) (22) database collects experimental and predicted information associated with the interactions of protein pairs in a given cell context via calculating the combined score of PPIs. The higher the combined score, the more reliable the PPIs are. In the present study, PPIs with a combined score $>0.4$ were considered to be significant. Thus, the DEGs were mapped into PPIs and a PPI network was then constructed based on the information of the STRING database.

\section{Results}

DEG analysis. Following data processing, the data was normalized for subsequent analysis. A total of 265 DEGs were obtained using the limma package in fracture hematoma tissues from old rats with rigid fixation compared with fracture hematoma tissues from old rats with semi-rigid fixation, and included 158 upregulated and 107 downregulated genes. For example, chemokine (C-X-C motif) ligand 12 (Cxcl12), chemokine (C-C motif) ligand 9 (Cc19) and matrix metallopeptidase 9 (mmp9) were upregulated, while $\operatorname{Tgf} \beta 1$, serpin peptidase inhibitor, clade $\mathrm{E}$ (nexin, plasminogen activator inhibitor type 1), member 1 (serpine1) and angiotensin II receptor, type 1a (Agtrla) were downregulated. The heat map plot of DEGs derived from hierarchical clustering analysis is shown in Fig. 1.

GO enrichment analysis. In order to understand the function of these DEGs, GO enrichment analysis was performed for DEGs. Table I displays the top 20 most significant GO-BPs, and the most overrepresented GO terms of these DEGs were found to be associated with the extracellular region, positive regulation of locomotion, response to external stimulus, positive regulation of cell migration and response to wounding.

PPI network analysis. Based on the information of the STRING database, the PPI network of DEGs was constructed with 153 nodes and 302 edges (Fig. 2). Additionally, the node degree distribution displayed that this PPI network was a scale-free network (Fig. 3). The biggest characteristic of the scale-free network was that a small number of nodes had higher degrees while the majority of the nodes had lower degrees, indicating that nodes with higher degrees may be important in network connectivity. Therefore, the nodes Tgf $\beta 1$ (degree, 20), Cxcl12 (degree, 19), Ccl9 (degree, 18), mmp9 (degree, 17), kinase insert domain receptor (degree, $15)$, platelet derived grow th factor receptor, $\beta$ polypeptide (degree, 14), serpinel (degree, 14), cadherin 2 (degree, 13), peroxisome proliferator-activated receptor $\gamma$ (degree, 13) and Agtrla (degree, 12) were hub proteins in the PPI network (Table II).

\section{Discussion}

The early phase of bone healing is likely to be sensitive to the conditions of mechanical loading (23). In the present study, a bioinformatics approach was used to identify key genes influenced in fixation stability from fracture hematoma tissues harvested 3 days post-osteotomy. Upregulated genes, 
including Cxcl12 and mmp9, and downregulated genes, such as Tgf $\beta 1$ and serpine1, were identified as hub nodes and appeared to be important in the process of fracture healing associated with fixation stability.

The fracture healing process may be divided into three stages: Acute inflammation, repair and remodeling (24). In the present study, upregulated genes, including Cxcl12 and mmp9, were key inflammatory cytokines and may be involved in the initial inflammatory response as the first step of fracture healing. The chemokine Cxcl12 has been identified to regulate the inflammatory response associated with the healing process (25). Furthermore, it is an important contributor to bone marrow MSC homing and localization within the bone marrow (26). Grassi et al (27) also indicated that Cxcl12 was involved in a number of inflammatory pathologies and was crucial in the regulation of osteoclast differentiation and function. In addition, mmp9 has been confirmed to have effects on skeletal cell differentiation during fracture healing via regulation of the inflammatory response and the inflammatory cell distribution (28). Furthermore, Beamer et al (12) demonstrated that mmp9 was implicated in the regulation of chondrogenic and osteogenic cell differentiation during the early stages of bone repair. Notably, Wang et al (29) indicated that the mechanical environment affected the inflammatory response and influenced skeletal cell differentiation during bone repair via the regulation of mmp9. Therefore, the results of the present study imply that fixation stability may induce an inflammatory response during fracture healing via the regulation of $\mathrm{Cxcl} 12$ and $\mathrm{mmp} 9$.

Tgf $\beta 1$ was identified as a hub node in the PPI network with the highest degree. It is a member of the transforming growth factor- $\beta$ family and functions as a regulatory protein involved in bone remodeling during the fracture healing process (13). Furthermore, Tgf $\beta 1$ is thought to induce the migration of bone mesenchymal stem cells and to regulate the coordination of bone resorption and subsequent bone formation (30). Additionally, it has also been found to be involved in fracture healing through regulation of the activation and differentiation of osteoblasts and osteoclasts (31). $\operatorname{Tgf} \beta 1$ induces the production of extracellular bone matrix proteins, including alkaline phosphatase, collagen, osteonectin, osteopontin and proteoglycans $(32,33)$, and can regulate different cell types implicated in bone turnover and fracture healing (34). Therefore, $\operatorname{Tgf} \beta 1$ may be important in the regulation of bone remodeling during the fracture healing process. However, in the present study, $\operatorname{Tgf} \beta 1$ was downregulated in fracture hematoma tissues from old rats with rigid fixation compared with fracture hematoma tissues from old rats with semi-rigid fixation, implying that rigid fixation may influence bone remodeling during the fracture healing process by the downregulation of $\operatorname{Tgf} \beta 1$.

Furthermore, serpine1 was identified as another downregulated hub node. Serpine1 [also known as plasminogen activator inhibitor-1 (PAI-1)], as a component of the fibrinolytic system, is the principal inhibitor of plasminogen activators (35). It has been shown to have various functions, including regulation of extracellular matrix (ECM) degradation and cell migration (36). Tamura et al (37) confirmed that PAI-1 suppressed the mRNA expression levels of runt-related transcription factor 2 and type I collagen in primary
Table I. Top 20 most significant pathways.

\begin{tabular}{lll}
\hline GO-ID & \multicolumn{1}{c}{ Description } & $\begin{array}{c}\text { Corrected } \\
\text { P-value }\end{array}$ \\
\hline 44421 & Extracellular region part & $2.49 \times 10^{-12}$ \\
40017 & Positive regulation of locomotion & $5.06 \times 10^{-9}$ \\
9605 & Response to external stimulus & $5.06 \times 10^{-10}$ \\
30335 & Positive regulation of cell migration & $1.02 \times 10^{-9}$ \\
9611 & Response to wounding & $1.04 \times 10^{-9}$ \\
5576 & Extracellular region & $1.53 \times 10^{-9}$ \\
51272 & Positive regulation of cellular & $1.80 \times 10^{-9}$ \\
& component movement & \\
42221 & Response to chemical stimulus & $1.85 \times 10^{-9}$ \\
32879 & Regulation of localization & $3.69 \times 10^{-9}$ \\
5615 & Extracellular space & $6.92 \times 10^{-9}$ \\
6954 & Inflammatory response & $1.25 \times 10^{-8}$ \\
32502 & Developmental process & $2.14 \times 10^{-8}$ \\
30334 & Regulation of cell migration & $2.27 \times 10^{-8}$ \\
42330 & Taxis & $2.27 \times 10^{-8}$ \\
6935 & Chemotaxis & $2.27 \times 10^{-8}$ \\
7275 & Multicellular organismal development & $2.40 \times 10^{-8}$ \\
31012 & Extracellular matrix & $3.70 \times 10^{-8}$ \\
40011 & Locomotion & $4.00 \times 10^{-8}$ \\
48731 & System development & $5.95 \times 10^{-8}$ \\
8009 & Chemokine activity & $7.92 \times 10^{-8}$ \\
& &
\end{tabular}

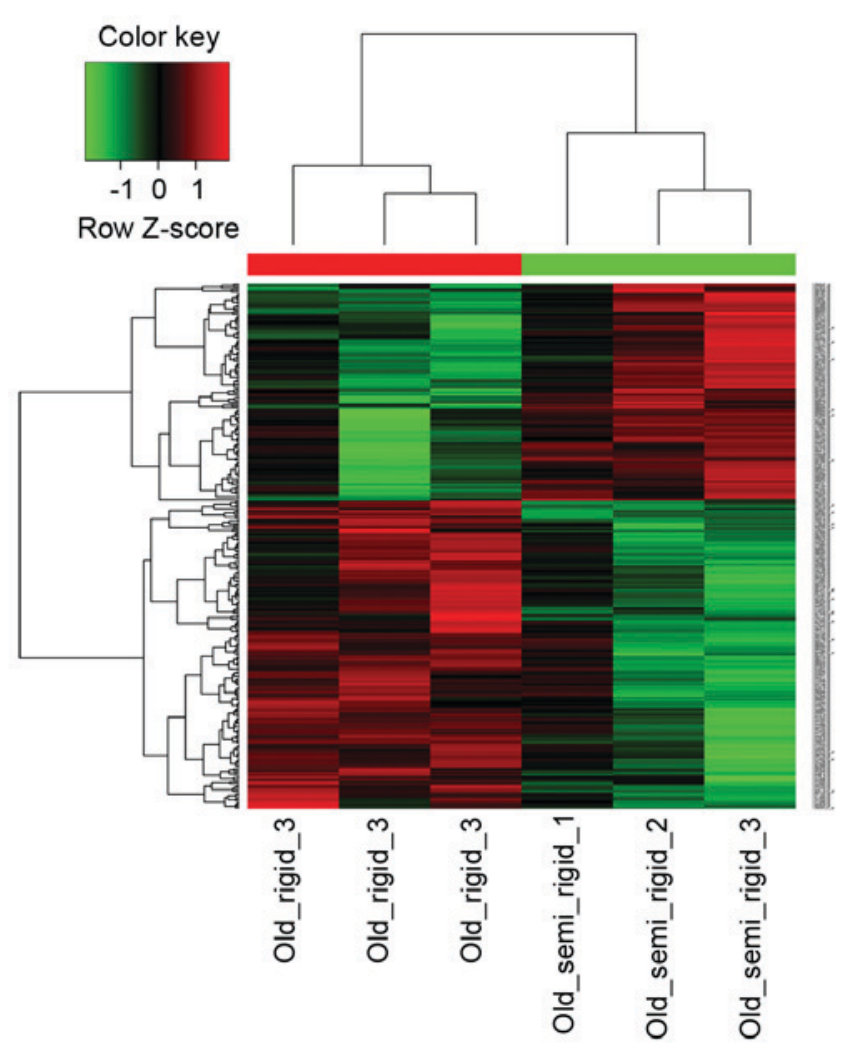

Figure 1. Heatmap plot of differentially expressed genes across old samples. The labels on the abscissa axis below the plot represent samples, and the markings above the plot represent the clustering of samples. The markings on the longitudinal axis represent the clustering of differentially expressed genes. Red represents high expression and green represents low expression. 
Table II. Genes with degree $\geq 10$.

\begin{tabular}{llcc}
\hline Gene & \multicolumn{1}{c}{ Name } & Log FC & Degree \\
\hline Tgf $\beta 1$ & Transforming growth factor, $\beta$ 1 & -1.567666199 & 20 \\
Cxcl12 & Chemokine (C-X-C motif) ligand 12 & 0.8016909 & 19 \\
Cc19 & Chemokine (C-C motif) ligand 9 & 0.588012021 & 18 \\
Mmp9 & Matrix metallopeptidase 9 & 1.752023742 & 17 \\
Kdr & Kinase insert domain receptor & 0.846463992 & 15 \\
Pdgfrb & Platelet derived growth factor receptor, $\beta$ polypeptide & 0.906423663 & 14 \\
Serpine1 & Serpin peptidase inhibitor, clade E (nexin, plasminogen & -1.17741059 & 14 \\
& activator inhibitor type 1), member 1 & & \\
Cdh2 & Cadherin 2 & 0.691787895 & 13 \\
Pparg & Peroxisome proliferator-activated receptor $\gamma$ & 0.78658078 & 13 \\
Agtr1a & Angiotensin II receptor, type 1a & -0.7331974 & 12 \\
Ednra & Endothelin receptor type A & 0.637602954 & 11 \\
Ccl20 & Chemokine (C-C motif) ligand 20 & 0.807308765 & 10 \\
Itgam & Integrin, $\alpha$ M & -1.252174861 & 10 \\
Nt5e & 5' nucleotidase, ecto & -0.831568237 & 10
\end{tabular}

FC, fold change.

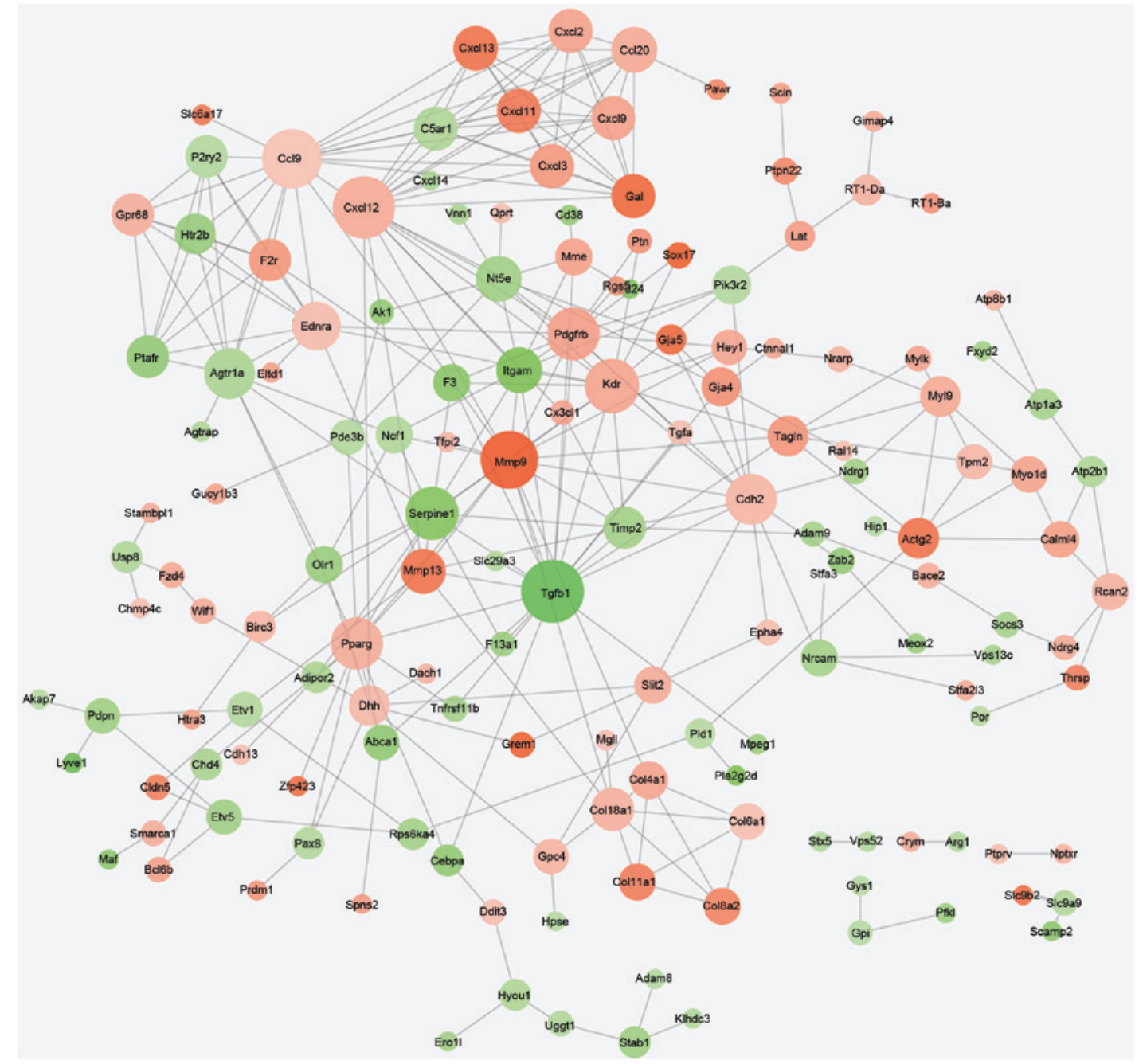

Figure 2. Protein-protein interaction network of differentially expressed genes. Node size reflects node degree where a larger size reflects a larger degree. Node color reflects fold-change values of the gene; red nodes are upregulated and green nodes are downregulated genes. The grey lines represent the interaction between genes. 


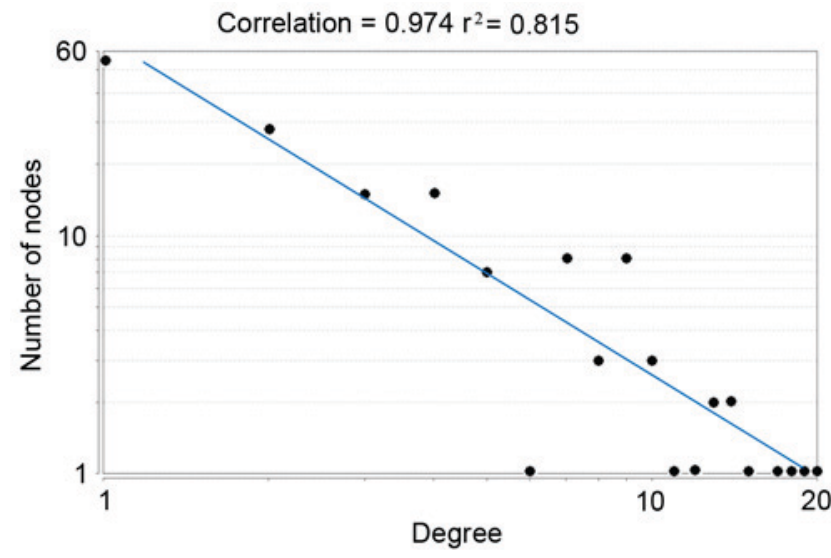

Figure 3. Node degree distribution. The blue line is a fitted power law distribution line. Black data points represent differentially expressed genes.

osteoblasts from mouse calvaria, suggesting that PAI-1 may suppress osteoblast differentiation during the bone repair process. In addition, serpinel has been reported to be important in the regulation of fracture callus size, cartilage formation and resorption during bone fracture repair (38). Mao et al (24) also demonstrated that PAI-1 may be involved in ECM remodeling and thus be crucial in the bone repair process in patients with diabetes. It may thus be speculated that serpine1 is important in bone fracture repair. In addition, Mao et al (24) also demonstrated that PAI-1 deficiency attenuated diabetic impaired bone repair in patients with diabetes. In the present study, serpinel was downregulated in fracture hematoma tissues with rigid fixation when compared with those with semi-rigid fixation, indicating that suppression of serpinel may promote bone repair following treatment using rigid fixation.

In conclusion, the observations of the present study indicate that fixation stability may influence the fracture healing process, and that important DEGs in fracture hematoma, including Cxcl12, mmp9, Tgf $\beta 1$ and serpine1 may be important in this process. The present observations shed new light on the molecular mechanism of fracture healing and have implications for future research. However, the small sample size and lack of experimental validation were limitations in the present study. To further validate the results, it would be prudent to perform the same analysis on a group of young rats and compare the two gene sets for common DEGs upon fixation rigidity in young and old rats.

\section{References}

1. Einhorn TA: The cell and molecular biology of fracture healing. Clin Orthop Relat Res (355 Suppl): S7-S21, 1998.

2. Dimitriou R, Tsiridis E and Giannoudis PV: Current concepts of molecular aspects of bone healing. Injury 36: 1392-1404, 2005.

3. Dimitriou R, Jones E, McGonagle D and Giannoudis PV: Bone regeneration: Current concepts and future directions. BMC Med 9: 66, 2011.

4. Ferguson C, Alpern E, Miclau T and Helms JA: Does adult fracture repair recapitulate embryonic skeletal formation? Mech Dev 87: 57-66, 1999.

5. Strube P, Sentuerk U, Riha T, Kaspar K, Mueller M, Kasper G, Matziolis G, Duda GN and Perka C: Influence of age and mechanical stability on bone defect healing: Age reverses mechanical effects. Bone 42: 758-764, 2008.
6. Mehta M, Strube P, Peters A, Perka C, Hutmacher D, Fratzl P and Duda GN: Influences of age and mechanical stability on volume, microstructure, and mineralization of the fracture callus during bone healing: Is osteoclast activity the key to age-related impaired healing? Bone 47: 219-228, 2010.

7. Epari DR, Kassi JP, Schell H and Duda GN: Timely fracture-healing requires optimization of axial fixation stability. J Bone Joint Surg 89: 1575-1585, 2007.

8. Ranganathan SI, Ferrari M and Decuzzi P: Design maps for scaffold constructs in bone regeneration. Biomed Microdevices 15: 1005-1013, 2013.

9. Kolar P, Schmidt-Bleek K, Schell H, Gaber T, Toben D, Schmidmaier G, Perka C, Buttgereit F and Duda GN: The early fracture hematoma and its potential role in fracture healing. Tissue Eng Part B Rev 16: 427-434, 2010.

10. Oe K, Miwa M, Sakai Y, Lee SY, Kuroda R and Kurosaka M: An in vitro study demonstrating that haematomas found at the site of human fractures contain progenitor cells with multilineage capacity. J Bone Joint Surg Br 89: 133-138, 2007.

11. Street J, Winter D, Wang JH, Wakai A, McGuinness A and Redmond HP: Is human fracture hematoma inherently angiogenic? Clin Orthop Relat Res 378: 224-237, 2000.

12. Beamer B, Hettrich C and Lane J: Vascular endothelial growth factor: An essential component of angiogenesis and fracture healing. HSS J 6: 85-94, 2010.

13. Sarahrudi K, Thomas A, Mousavi M, Kaiser G, Köttstorfer J, Kecht M, Hajdu S and Aharinejad S: Elevated transforming growth factor-beta 1 (TGF- $\beta 1$ ) levels in human fracture healing. Injury 42: 833-837, 2011.

14. Ode A, Duda GN, Geissler S, Pauly S, Ode JE, Perka C and Strube P: Interaction of age and mechanical stability on bone defect healing: An early transcriptional analysis of fracture hematoma in rat. PLoS One 9: e106462, 2014

15. Gautier L, Cope L, Bolstad BM and Irizarry RA: Affy-analysis of Affymetrix GeneChip data at the probe level. Bioinformatics 20: 307-315, 2004

16. Smyth GK: Limma: Linear models for microarray data. In: Bioinformatics and Computational Biology Solutions using $\mathrm{R}$ and Bioconductor. Gentleman R, Carey VJ, Huber W, Irizarry RA and Dudoit S (eds). Springer, New York, pp397-420, 2005.

17. Wang L, Cao C, Ma Q, Zeng Q, Wang H, Cheng Z, Zhu G, Qi J, Ma H, Nian H and Wang Y: RNA-seq analyses of multiple meristems of soybean: Novel and alternative transcripts, evolutionary and functional implications. BMC Plant Biol 14: 169, 2014.

18. Ashburner M, Ball CA, Blake JA, Botstein D, Butler H, Cherry JM, Davis AP, Dolinski K, Dwight SS, Eppig JT, et al: Gene ontology: Tool for the unification of biology. The Gene Ontology Consortium. Nat Genet 25: 25-29, 2000.

19. Maere S, Heymans K and Kuiper M: BiNGO: A Cytoscape plugin to assess overrepresentation of Gene Ontology categories in biological networks. Bioinformatics 21: 3448-3449, 2005.

20. Smoot ME, Ono K, Ruscheinski J, Wang PL and Ideker T: Cytoscape 2.8: New features for data integration and network visualization. Bioinformatics 27: 431-432, 2011.

21. Benjamini Y and Hochberg Y: Controlling the false discovery rate: A practical and powerful approach to multiple testing. J R Stat Soc Series B 57: 289-300, 1995.

22. Franceschini A, Szklarczyk D, Frankild S, Kuhn M, Simonovic M, Roth A, Lin J, Minguez P, Bork P, von Mering C and Jensen LJ: STRING v9. 1: Protein-protein interaction networks, with increased coverage and integration. Nucleic Acids Res 41: D808-D815, 2013

23. Klein P, Schell H, Streitparth F, Heller M, Kassi JP, Kandziora F, Bragulla H, Haas NP and Duda GN: The initial phase of fracture healing is specifically sensitive to mechanical conditions. J Orthop Res 21: 662-669, 2003.

24. Mao L, Kawao N, Tamura Y, Okumoto K, Okada K, Yano M, Matsuo $\mathrm{O}$ and Kaji $\mathrm{H}$ : Plasminogen activator inhibitor-1 is involved in impaired bone repair associated with diabetes in female mice. PLoS One 9: e92686, 2014.

25. Galliera E, Corsi M and Banfi G: Platelet rich plasma therapy: Inflammatory molecules involved in tissue healing. J Biol Regul Homeost Agents 26 (2 Suppl 1): 35S-42S, 2011.

26. Honczarenko M, Le Y, Swierkowski M, Ghiran I, Glodek AM and Silberstein LE: Human bone marrow stromal cells express a distinct set of biologically functional chemokine receptors. Stem cells 24: 1030-1041, 2006. 
27. Grassi F, Cristino S, Toneguzzi S, Piacentini A, Facchini A and Lisignoli G: CXCL12 chemokine up-regulates bone resorption and MMP-9 release by human osteoclasts: CXCL12 levels are increased in synovial and bone tissue of rheumatoid arthritis patients. J Cell Physiol 199: 244-251, 2004.

28. Pape HC, Marcucio R, Humphrey C, Colnot C, Knobe M and Harvey EJ: Trauma-induced inflammation and fracture healing. J Orthop Trauma 24: 522-525, 2010.

29. Wang X, Yu YY, Lieu S, Yang F, Lang J, Lu C, Werb Z, Hu D, Miclau T, Marcucio R and Colnot C: MMP9 regulates the cellular response to inflammation after skeletal injury. Bone 52: $111-119,2013$

30. Tang Y, Wu X, Lei W, Pang L, Wan C, Shi Z, Zhao L, Nagy TR, Peng X, Hu J, et al: TGF-betal-induced migration of bone mesenchymal stem cells couples bone resorption with formation. Nat Med 15: 757-765, 2009.

31. Estai MA, Suhaimi F, Das S, Shuid AN, Mohamed Z and Soelaiman IN: Expression of TGF- $\beta 1$ in the blood during fracture repair in an estrogen-deficient rat model. Clinics (Sao Paulo) 66: 2113-2119, 2011.

32. D'Amelio P, Cristofaro MA, Grimaldi A, Ravazzoli M, Pluviano F, Grosso E, Pescarmona GP and Isaia GC: The role of circulating bone cell precursors in fracture healing. Calcif Tissue Int 86: 463-469, 2010.
33. Sandblrg MM, Aro HT and Vuorio EI: Gene expression during bone repair. Clin Orthop Relat Res: 292-312, 1993.

34. Vo TN, Kasper FK and Mikos AG: Strategies for controlled delivery of growth factors and cells for bone regeneration. Adv Drug Deliv Rev 64: 1292-1309, 2012.

35. Gils A and Declerck PJ: Plasminogen activators inhibitors. In: Plasminogen: Structure, Activation and Regulation. Waisman DM (ed). Springer, New York, pp47-66, 2003.

36. Declerck PJ and Gils A: Three decades of research on plasminogen activator inhibitor-1: A multifaceted serpin. Semin Thromb Hemost 39: 356-364, 2013.

37. Tamura Y, Kawao N, Okada K, Yano M, Okumoto K, Matsuo O and Kaji $\mathrm{H}$ : Plasminogen activator inhibitor-1 is involved in streptozotocin-induced bone loss in female mice. Diabetes 62: 3170-3179, 2013.

38. Rundle $\mathrm{CH}$, Wang $\mathrm{X}$, Wergedal JE, Mohan $\mathrm{S}$ and Lau KH: Fracture healing in mice deficient in plasminogen activator inhibitor-1. Calcif Tissue Int 83: 276-284, 2008. 